\title{
Can we safely use a wait and see approach for patients with recent onset atrial fibrillation?
}

Saahil Vij, MD*; Amy Burton, MD*; Heather Murray, MD, MSc, FRCP(C) ${ }^{\dagger}$; Melanie Walker, $\mathrm{PhD}^{\dagger}$

\begin{abstract}
link: https://www.nejm.org/doi/full/10.1056/ NEJMoa1900353

Full citation: Pluymaekers NA, Dudink EA, Luermans JG, et al. Early or delayed cardioversion in recent-onset atrial fibrillation. $N$ Engl $J$ Med 2019;380(16):14997-508.
\end{abstract}

Article type: Therapy

Ratings: Methods - 4/5 Usefulness - 2/5

\section{INTRODUCTION}

\section{Background}

Patients with recent onset, stable, and symptomatic atrial fibrillation in the emergency department (ED) traditionally neccessitate immediate cardioversion, but a portion will convert without any intervention.

\section{Objectives}

To determine whether a wait-and-see approach was noninferior to early cardioversion in patients presenting with recent onset, stable, and symptomatic atrial fibrillation.

\section{STRUCTURED METHODS}

\section{Design}

Multicentred, randomized, open-label, and non-inferiority trial.

\section{Setting}

Fifteen cardiology departments in the Netherlands, coordinated by the Maastricht University Medical Centre.

\section{Intervention}

Cardioversion at initial visit v. rate control and return visit for cardioversion within 48 hours of symptom onset.

\section{Eligibility criteria}

Patients $\geq 18$ years of age presenting to the ED between 2014 and 2018 with hemodynamically stable, symptomatic, and recent onset atrial fibrillation.

\section{Outcomes}

Primary endpoint included sinus rhythm on electrocardiogram (ECG) at the four-week follow-up visit. Secondary endpoints included length of stay of the primary visit, subsequent visits related to atrial fibrillation, cardiovascular complications, and time to recurrence of atrial fibrillation.

\section{MAIN RESULTS}

A total of 437 patients were randomized: 218 to the delayed cardioversion group, and 219 to the early cardioversion group. In the early cardioversion group, 94\% of the patients were in sinus rhythm at the four-week visit, with $16 \%$ spontaneously converting and $78 \%$ undergoing electrical or chemical cardioversion at their initial ED visit. In the delayed cardioversion group, $91 \%$ of the patients were in sinus rhythm at the four-week visit, with $69 \%$ having converted spontaneously within 48 hours. Of those in the delayed group, $83 \%$ received rate control agents during the initial ED visit. The difference of $2.9 \%$ met the pre-specified non-

From the *Emergency Medicine, Queen's University, Kingston, ON; and the ${ }^{\dagger}$ Department of Emergency Medicine, Queen's University, Kingston, ON.

Correspondence to: Dr. Saahil Vij, Sunnybrook Hospital, Department of Emergency Medicine, 2075 Bayview Avenue, C753, Toronto, Ontario M4N 3M5; Email: saahil.vij@medportal.ca

(c) Canadian Association of Emergency Physicians 
inferiority margin of $10 \%$ (95\% confidence interval [CI] -8.2 to $2.2, p=0.005$ for non-inferiority). There were no significant differences between the two groups or in terms of cardiovascular complication rates at the four-week visit.

\section{APPRAISAL}

\section{Strengths}

- Clinically relevant PICO (Population, Intervention, Comparison, Outcome) question

- Large multicentred, randomized control (noninferiority) trial

- Balance of baseline characteristics across treatment groups

\section{Limitations}

- Primary outcome not explicitly correlated with patient preferences or ED clinical priorities

- 48-hour rhythm status likely of more interest to patient and a more relevant primary outcome

- ED cardioversion by cardiology and anesthesia and use of flecainide not consistent with Canadian ED practices $^{2}$

- Non-blinded trial; possibility for misclassification bias

- Unclear if outcome assessors were blinded to ECGs during central assessment; risk of detection bias

- Primary analysis was not a true intention to treat; patients who withdrew consent or were lost to follow-up were excluded

- Unclear how many patients refused enrolment as most sites did not provide screening logs

- Adverse outcome list was not exhaustive of all potential embolic phenomena

- Pending data from planned longer-term follow-up relevant to ED specific priorities

\section{CONTEXT}

Current Canadian emergency medicine practice guidelines recommend immediate cardioversion for symptomatic, recent onset atrial fibrillation. ${ }^{1}$ The goal of this paper was to consider if the current management of this common presentation could be safely changed to reduce overtreatment and decrease stress on EDs; however, arranging for two visits within 48 hours of symptom onset (potentially within 12 hours) is not convenient for patients or the hospital. Future research could assess patient characteristics that are associated with a likelihood to spontaneously cardiovert, a cost analysis, and other clinical repercussions between delayed and early cardioversion.

\section{BOTTOM LINE}

This trial initiates a discussion surrounding our management of symptomatic, recent onset atrial fibrillation. The early cardioversion arm was cardioverted at their initial visit, and the delayed cardioversion arm was rate controlled and reassessed within 48 hours of symptom onset with cardioversion if still in atrial fibrillation. The delayed arm was noninferior for a primary outcome of sinus rhythm at four weeks. However, this primary outcome may not align with the priorities of physicians or patients, and return visits within 48 hours are not ideal for resources or patient satisfaction. Pending data from this study may help clarify this clinical question.

Keywords: Emergency medicine, cardiac disease, arrhythmia, evidence-based medicine

\section{REFERENCES}

1. Andrade JG, Verma A, Mitchell LB, et al. 2018 Focused update of the Canadian Cardiovascular Society guidelines for the management of atrial fibrillation. Can 7 Cardiol 2018;34(11):1371-92.

2. Stiell EG, Scheuermeyer FX, Vadeboncoeur A, et al. CAEP Position Statement: CAEP Acute Atrial Fibrillation/Flutter Best Practices Checklist. C7EM 2018;20(3):334-42. 